\title{
Making Sense of Trajectory Data in Indoor Spaces
}

\author{
Thor Prentow \\ Dept. of Computer Science \\ Aarhus University, DK \\ prentow@cs.au.dk
}

\author{
Andreas Thom \\ Dept. of Computer Science \\ University of Münster, DE \\ andreas.thom@uni-muenster.de
}

\author{
Henrik Blunck \\ Dept. of Computer Science \\ Aarhus University, DK \\ blunck@cs.au.dk
}

\author{
Jan Vahrenhold \\ Dept. of Computer Science \\ University of Münster, DE \\ jan.vahrenhold@uni-muenster.de
}

\begin{abstract}
The increasing prevalence of positioning and tracking systems has helped simplify tracking large amounts of, e.g., people moving through buildings or cars traveling on roads, over long periods of time. However, technical limitations of positioning algorithms and traditional sensing infrastructures are likely, especially indoors, to induce errors and biases in the resulting data. In particular, the resulting motion trajectories often do not conform perfectly to the underlying route network. As a consequence, analyses of trajectory sets are impeded by these phenomena, as it becomes hard to identify which route was taken in a particular travel instance or whether two travel instances followed the same route. In this paper, we present a bootstrapping approach and several algorithms to mitigate error biases and related phenomena, focusing on indoor scenarios. In particular, we are able to estimate and iteratively refine an underlying route network from a set of motion trajectories. Secondly, we represent subtrajectories, i.e., movements on individual elements of the route network, by their median subtrajectory. The resulting aggregated and cleaned-up data set facilitates using further, domain-specific analysis tools. Additionally, it allows to predict the locally occurring expected positioning error biases. This in turn allows improved positioning, e.g., for real-time navigation assistance scenarios. We evaluate the proposed methods using trajectory data from employees at a large hospital complex. In particular, we show that we can reconstruct the hospital's route network accurately, and that we can furthermore extract median subtrajectories for almost all individual corridors. Finally, we illustrate that median trajectories deliver useful deviation maps to learn, and correct for, the expected local biases in positioning.
\end{abstract}

\section{INTRODUCTION}

Advances in positioning technologies have made it easy to automatically and continually track entities moving, e.g., throughout large building complexes or on road networks over long periods of time. Exploiting already existing infrastructures, such as Wi-Fi and GSM networks or the global positioning systems allows for easily-deployable, low-maintenance positioning both in indoor and outdoor scenarios. The positioning data obtained by these systems, however, suffers from various kinds of noise and inaccuracies that may hamper further analyses of the collected position traces if not accounted for. On top of this, position estimates can be influenced by building layout, movement of people and equipment, as well as changes in Wi-Fi infrastructure and variances in $\mathrm{Wi}-\mathrm{Fi}$ emission signal strength, e.g., for the sake of load balancing, causing outliers in position estimates with rather large errors [16]. There exist dedicated hardware/software solutions, e.g., Ekahau ${ }^{\mathrm{TM}}$ active RFID tags, that considerably improve the position estimates. Requiring users to install software and/or use dedicated hardware, however, is infeasible in many applications, both for ease-of-maintenance and for privacy reasons. Examples include the anonymized analysis of motion patterns in a hospital
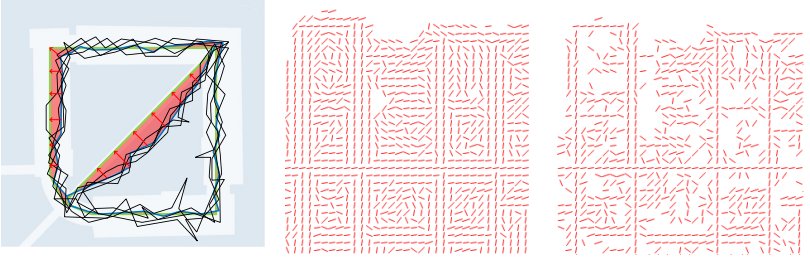

Fig. 1: Left: Input traces (black), ground-truth route network (green), median trajectories (blue) and deviation maps (red). Right: Majority axis-angle for ach grid cell, as computed in route network reconstruction, before (middle) and after (right) removing insignificant bins.

(which is our motivating example, see also Section IV for a discussion of the positioning methods employed there) or the retrospective assessment of the layout of booths in a fair and the analysis of the induced motion patterns of visitors and resulting hotspots in the layout.

In this paper, we present methods for analysis of trajectory data and for preparing trajectory data for further analyses. These methods focus on inferring information about the location system's performance and bias, and potentially also about the surroundings in which the users move. To handle the most general case, we assume that the underlying route network is unknown (RNU scenario). Such scenarios include fairs or other public events where the route network is induced by temporary booths or exhibits. For many building complexes, appropriate digital route network models are neither given nor easily obtainable [22], [16] as well, so that also here the proposed means for route network construction serves to obtain the route network or to facilitate or sanity-check the construction by other means. Our approach is further motivated by outdoor cases, where the analysis goals include to infer an implicit route network, e.g., when analyzing preferred motion patterns in open outdoor areas. In an RNU scenario, our algorithm supports all of the three deliverables detailed below: 1) Route network reconstruction, 2) Deviation maps, and 3) Trajectory cleaning.

1) Route network reconstruction: As a first step for handling an RNU scenario, our algorithm computes an approximation of the underlying route network from the input trajectories; the initial process is illustrated in Figure 1 (middle and right) and an example result, for the hospital chosen as evaluation environment, is shown in Figure 7. This allows to provide the deliverables detailed further below even for cases where the underlying route network is not known a priori. In these cases, the reconstructed route network may be of independent interest, because manually building such a network may be prohibitively time-consuming [1], [16]. Even when the route 
network is known, a network reconstructed from collected trajectories contains additional information, as it may reflect the route segments actually used better than the model known a priori. Furthermore, such a network is better suited to detect implicit route segments, e.g., in large open spaces.

2) Deviation maps: The accuracy of indoor positioning systems is influenced locally by static elements, e.g., the architecture of a building. Hence, positioning in or around buildings often has static local biases, distorting the positioning estimate in a specific direction. By aggregating all subtrajectories that correspond to a particular segment of the (approximate or known) route network into an average [18] or median trajectory [6] we compute a representative of the estimated movement pattern along this segment; see the blue trajectory in Figure 1 (left). Using a route network (given or reconstructed) and these representative trajectories, we can determine the static biases in the positioning system at specific locations and along paths typically used; see the red areas in Figure 1 (left). Comparing the representative trajectory ${ }^{1}$ to the ground-truth or approximate location of route segments, we can estimate the expected location deviation, or bias. The resulting deviation map can be used to correct for this bias when estimating locations or to prioritize improvements on the positioning system.

3) Trajectory cleaning: Trajectories from inaccurate positioning systems may contain position outliers, i.e., position estimates that are extraordinarily far from the actual locations; see the bottom part of Figure 1 (left). Snapping each individual trajectory to the representatives of the trajectory's segments, one obtains an outlier-free trajectory, which represents the route actually taken. The snapping eases further analyses, because trajectories following the same segments of the route network will have an identical geometry. This greatly facilitates, e.g., pattern discovery [13] or the comparison of routes. Another cleaning option would be to consider the average or median trajectory as a reference and to declare a position estimate as an outlier if its distance to the reference under a geometric similarity measure such as the Hausdorff or Fréchet distance [7], [18] is above a given threshold.

In addition to these main deliverables, the output of our algorithm provides additional deliverables to at least two other relevant types of analyses.

Outlier movement detection: The representative trajectories computed for each segment of the route network may be used to detect outliers in movement patterns. By comparing collected position traces to the representative trajectory, we can determine if a particular movement is at least roughly in accordance with what has been seen before. This can be used, e.g., to detect that an obstacle has emerged in a building or road network, causing traversals to follow alternative paths.

Self-healing analysis: Deviation maps and representative trajectories can be used to improve the accuracy of snapping (parts of) trajectories to the route network. In this way, trajectory cleaning will improve over time as further trajectories are collected, as will the outlier movement detection.

\footnotetext{
${ }^{1}$ Because user orientation may block signals differently depending on the travel direction [3], we may need one reference trajectory per direction.
}

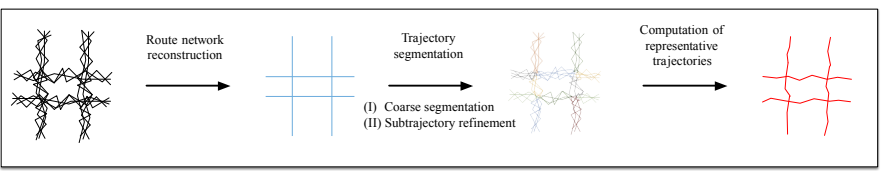

Fig. 2: Illustration of the processing pipeline showing the three main modules as well as examples of inputs and outputs.

\section{RELATED WORK}

For outdoor scenarios, various work has been published on route network reconstruction, mostly of car road networks [11], [5], [19]. Furthermore, also map matching strategies have been proposed for when route network and/or positioning information are given but are flawed in terms of accuracy and thus cannot be fully relied on [23], [20]. For reconstruction of indoor location models, such as route network models or floor plans, various approaches have been proposed. Many of these are formulated as solution to the simultaneous localization and mapping (SLAM) problem or borrow from such solutions. SLAM, originating in robotics, has often been formulated as the ultimate goal of truly autonomous robotic movement[10]. Recently, SLAM-like approaches have been proposed for floor plan reconstruction through observations by devices carried by humans. SmartSLAM [2] is based solely on various smartphone sensors. Du et al. [9] build depth camera models, Jiang et al. [15] rely on Wi-Fi fingerprints and user information, and Alzantot et al. [1] leverage additionally GPS and inertial sensors. Other approaches, e.g., Philipp et al.'s MapGENIE [21], employ foot-mounted sensors additional to smartphone installations. Ferris et al. proposed WiFi-SLAM [12], and Huang et al. [14] a variant of it that utilized techniques from GraphSLAM.

Most of the above approaches either require specific procedures to follow, specialized software/hardware installations, or active data sharing by the user. Such approaches are often infeasible in use cases like, e.g., tracking visitors in buildings or outdoor areas to better understand motion patterns. The remaining cited approaches do not detail how Wi-Fi signal measurements are obtained or by which devices and software installations these are processed. Somewhat in contrast, our approach investigates route network construction based on a minimally invasive, minimal-setup data collection, based solely on the natural movements of the investigated environments' users, and on locating these users solely by radio signals that their consumer devices emit without the explicit need to install dedicated on-device software [17]. We show furthermore, that despite our method's low requirements, they achieve a accuracy in, e.g., detection of route network intersections (and their location) which is comparable to results published for state of the art methods [11] evaluated in outdoor scenarios.

\section{METHODS}

We produce the deliverables of our methods through three main modules that are connected in a pipeline to process and analyze the input trajectory data, as illustrated in Figure 2.

\section{A. Route network reconstruction}

The route network reconstruction module analyzes the noisy trajectories in order to reconstruct the underlying route network which constrains the actual movements associated 

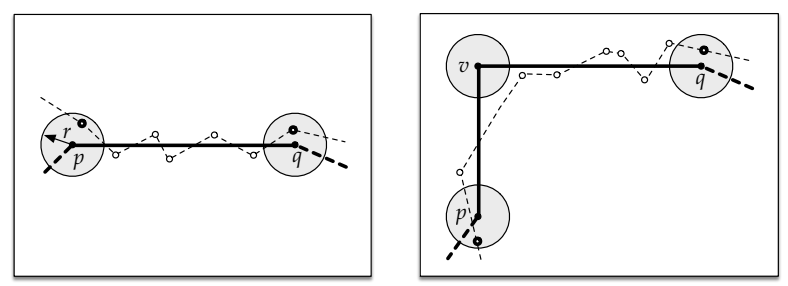

Fig. 3: Recognizing subtrajectories: correctly matching a corridor (left); missing an corridor endpoint $v$ (right).

with the collected trajectories. Specifically, the module finds intersection points and major segments of the network.

1) Rasterizing the trajectories: The area covered by the trajectories is subdivided into grid cells of size $\sigma$. In each cell the space of orientations is subdivided into $b$ bins, each representing an angle interval of $\frac{\pi}{b}$. Each grid cell holds $b$ counters, initially set to zero, that indicate how many trajectory segments cross this cell oriented along the $b$ main directions, respectively. For each cell the majority angle is determined, i.e., the bin with the highest count among the cell's bins. Then, bins with insignificant counts are removed, as they likely stem from noise or outliers in the position estimates. Bins are deemed insignificant if their count is lower than the average count across the bins for the same angle in the neighboring grid cells. Figure 1 illustrates the majority axis-angle, using $b=8$, before (middle) and after (right) removal of insignificant bins. For de-noising, recorded rapid position jumps within trajectories are removed beforehand-by filtering trajectory segments against an upper speed threshold $T_{d}$.

2) Identifying major travel directions: Next, the algorithm identifies maximal connected components of cells that are traversed in the same direction. To this end, on top of the grid a graph is constructed, where each grid cell is represented by a node, and where an edge is created for every two cells which are adjacent and have the same majority angle. Then the maximal connected components are computed, resembling maximal route segments with the same major direction. Each such component is the represented by a line segment. Following, the algorithm resets in each component the counters of the bins that resemble the majority angles to zero. This corresponds to removing the segments added to the route network from the raw-data grid. The algorithm then repeats the above procedure until no further non-trivial connected components can be found. The result of this process is a collection of line segments corresponding to (possibly intersecting) connected components. To ensure that the extracted network segments will intersect even if these are slightly shorter than the corresponding route segments in the underlying route network, we extend the former by up to $e$ meters in each direction iff this leads to a intersection of extracted segments. Finally, a line-segment intersection algorithm [4] computes all intersections and, hence, the topology of the route network.

We determined empirically suitable parameters, specific to the hospital environment, i.e., we choose $\sigma=3 \mathrm{~m}$, which corresponds to the average corridor width, $T_{d}=25 \mathrm{~m} / \mathrm{s}$ as an upper bound on the realistically occuring speeds in the hospital, and $e=10 \mathrm{~m}$, where good results were generally obtained for $e \geq 2 \sigma=6 \mathrm{~m}$.
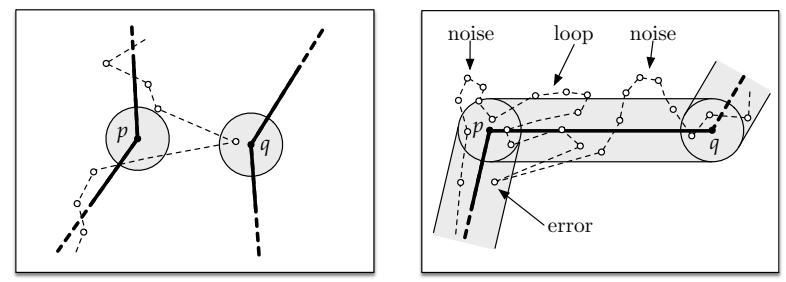

Fig. 4: Cleaning noisy data: outliers may lie close to intersection points (left), and subtrajectories may loop (right).

\section{B. Trajectory segmentation}

The purpose of the trajectory segmentation module, which is described in greater detail in the full version of this paper, is to match the trajectories to a route network. For this, the matching algorithm iteratively processes the input trajectories and subdivides each trajectory into a collection of subtrajectories that connect intersection points in the route graph. Each such subtrajectory is then considered individually and is aligned with the respective edges (corridors) in the route network. During this process, noise and erroneous data points are eliminated. To identify (coarse) subtrajectories, the algorithm traverses each given trajectory from its start point and tries to identify the intersection points $p$ of the route network close to, and thus relevant for, this actual trajectory, i.e., within a radius of $r$ meters (we used $r=12 \mathrm{~m}$ ) of a trajectory data point $x$. To properly handle, i.e., ignore, isolated outliers that lie close to an intersection point (see Figure 4), we also require the majority of the $\kappa$ data points preceding and succeeding $x$ to have one of the corridors adjacent to $p$ as their geometrically closest feature of the route network (we used $\kappa=5$ ). Identifying all relevant intersection points along the trajectory, allows for subdividing it parts delimited by data points matched to two consecutive relevant intersection points.

The algorithm may identify additional corridors, e.g., if these were filtered out initially in the reconstruction phase due only few trajectories passing along them, These corridors can be added to the route network and used for matching trajectories subsequently.

Next, in a refinement step, we aim to align the subtrajectories, produced as detailed above, to corridors in the network. Ideally, the two intersection points inducing a subtrajectory are endpoints of the same corridor, e.g., $p$ and $q$ in Figure 3 (left). Alternatively, the trajectory though may miss intermediate intersection points (Figure 3, right) or there may exist a yet undetected corridor between $p$ and $q$. To resolve both cases, first each data point is labeled with the closest feature (intersection point or corridor) of the route network. Outliers are then removed from this sequence in order to obtain a denoised sequence of route network elements actually traversed. Handling of outliers depends on whether they follow one corridor or jump between corridors, see Figure 4. Afterwards, the de-noised sequence for each subtrajectory processed is further simplified-by removing loops, which loop in short order between an intersection point and adjacent corridor segments, as shown in Figure 4 (right).

Ideally, the resulting subtrajectories are assigned labels of only two intersection points and the corridor between them. For more complex label sequences, a set of candidate corridors is extracted from the route network: For each data point of the 

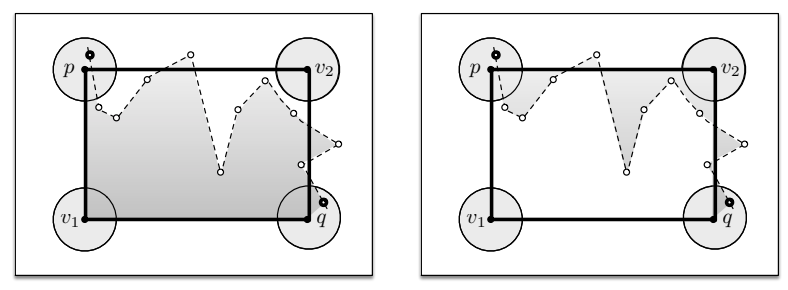

Fig. 5: Resolving ambiguities: Assessing path-corridor mappings by comparing enclosed areas.

subtrajectory, the closest corridor, irrespective of the distance, is computed and a subgraph of the route network that consists of these corridors and their endpoints is constructed. If in this graph no path from start to end point of the subtrajectory exists, the algorithm creates paths so that these augment the network with tentative corridor segments in only a minimal manner, as detailed in the full version of the paper.

Afterwards, if several candidate paths exist, we choose the one with the lowest assessment score, calculated as the area between the path and the subtrajectory, see Figure 5, times $(1+1 /(1+\sqrt{\omega})+\nabla)$, where $\omega$ is the number of data points assigned to tentatively created corridors, and $\nabla$ is the number of corridors for which there exists no witness data point, i.e. no data point having them as its closest corridor.

If the chosen path contains a corridor constructed as a tentative corridor while processing the current subtrajectory it is added to the full route network. We then simultaneously traverse the path and the subtrajectory and create maximal subsequences of data points assigned to the same corridor. Between these subsequences, we create splitting points corresponding to the intersection points between two consecutive corridors. Each element in the resulting final subdivision of the current subtrajectory corresponds to exactly one corridor. In case though that two consecutive elements are assigned to non-adjacent corridors, we mark these as an overly noisy part of the trajectory - and exclude it from further consideration when computing representative trajectories.

\section{Computation of representative trajectories}

The above phase produces an updated route network, but also assigns subtrajectories to a corridor in the network. For each corridor element we now aggregate the subtrajectories assigned to it into a single subtrajectory representing the movement in this corridor. This aggregation not only aids the visual perception of the resulting network but also facilitates various analyses, e.g., the computation of deviation maps.

To obtain representatives for movements assigned to a corridor an obvious candidate is their average trajectory [18]. This approach is computationally efficient and works well in scenarios with a regular layout. It is less suited when curved trajectories, or, more precisely, trajectories not following one major directions, are involved. In such situations, computing a median trajectory [6] is advantageous: This approach constructs the arrangement, i.e., the embedded graph, induced by the set of segments of all subtrajectories in question. It then assembles the median trajectory via traversing this graph, moving along all segments for which the same number of other segments lie on either side. Computing a median trajectory is computationally more involved but has two advantages.

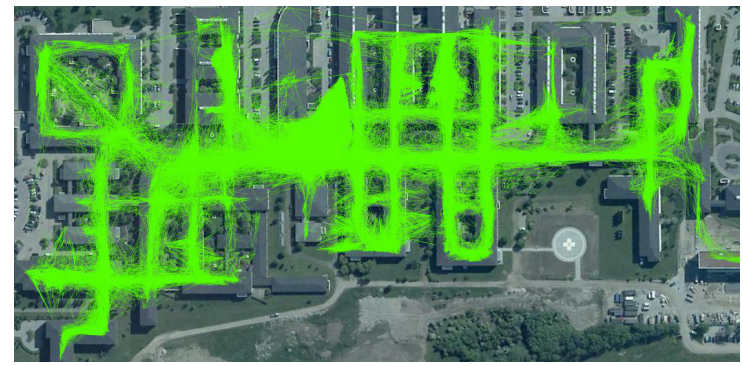

Fig. 6: The indoor trajectory dataset collected within the hospital. Map: Google, Aerodata International Surveys

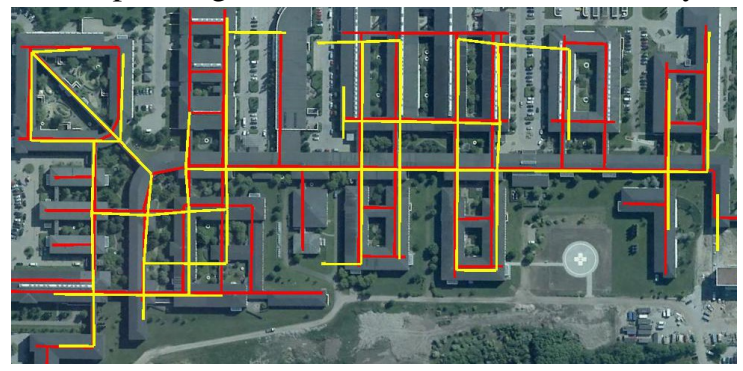

Fig. 7: Route Network: ground-truth (red) and reconstruction (yellow). Map: Google, Aerodata International Surveys

The first advantage is that, because local decisions are made, handling of curved (parts of) trajectories is improved. Secondly, it is more applicable for outlier movement detection, as described in the introduction: If an obstacle emerges on a path and roughly half of the trajectories pass on either side of this obstacle, the computed average trajectory will will pass right through that obstacle. In contrast, the median trajectory, consisting of segments of input trajectories, will pass on one of the sides of the obstacle. Generally speaking, this form of aggregation aims to ensure that the resulting movement representative is actually traversable within the route network.

\section{Evaluation}

Our evaluation of the proposed methods is based on a indoor trajectory dataset collected at a large hospital. The trajectories have been collected by employees at the hospital while performing their daily routines. The employees were given ten smart phones which were carried throughout their work shifts for ten days. The phones had software installed which performed Wi-Fi signal strength measurement scans about every two seconds. By pairing these measurements with knowledge of the access point locations at the hospital, we compute position estimates for each scan using the centroid lateration Wi-Fi positioning method [8], which we evalutated to yield a median accuracy of $15 \mathrm{~m}$ at the hospital [22]. The collected trajectory dataset, shown in Figure 6, contains 1.5 million position estimates.

\section{A. Route network reconstruction}

To evaluate the route network reconstruction, we apply it to the hospital data in Figure 6 visually compare, in Figure 7, its results (yellow) to the actual network as known from floor plans (red). A corresponding statistical evaluation is given in Table I which gives true and false positives as well as false negatives, both terms of number of instances, i.e. corridor segments, as well as in length in meter. Results are listed for different numbers $b$ of modeled corridor directions. We also 

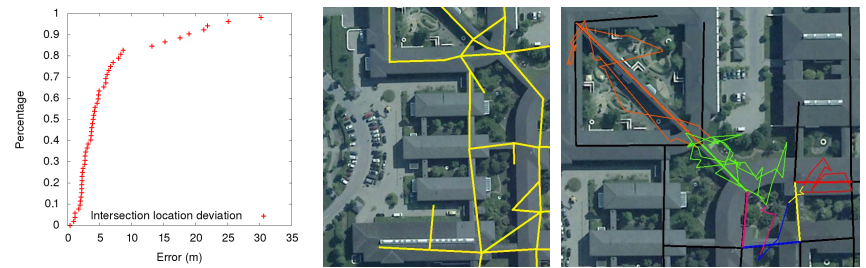

Fig. 8: Left: Cumulative error distribution of intersection placement. Middle: Reconstruction, using less conservative detection than in Figure 7. Right: A corridor segmentation for a trajectory part. Maps: Google, Aerodata International Surveys

\begin{tabular}{c||c|c|c||c|c|c||c|c|c||}
\multicolumn{1}{l||}{} & \multicolumn{3}{c||}{$b=8$} & \multicolumn{3}{c||}{$b=4$} & \multicolumn{3}{c||}{$b=16$} \\
& TP & FP & FN & TP & FP & FN & TP & FP & FN \\
\hline Count & 85 & 7 & 29 & 84 & 29 & 30 & 79 & 2 & 35 \\
\hline$m$ & 3000 & 200 & 1300 & 3200 & 870 & 1100 & 2400 & 90 & 1900
\end{tabular}

TABLE I: Accuracies of the route network reconstruction

varied of the grid cell size parameter $\sigma$, varying it from $5 \mathrm{~m}$ to $20 \mathrm{~m}$, but found its influence to be comparatively insignificant. Table I confirms the few false positives, i.e., reconstructed segments where no real corridor exists in the floor plans, many of which are caused by sudden erroneous jumps in the positioning. However, some also seem to be real (or virtual, resulting from strong access point reception) shortcuts between the buildings which are not represented in the floor plans.

The comparison of Figures 6 and 7 shows that corridors frequently traversed are reconstructed correctly. Missing reconstruction occurs mostly for short corridor segments close to the main corridors-which are thus hard to distinguish from (noisy) data from travels on the main corridors, or, more prominently, for corridors traversed only rarely. As such the reconstructed route network better represents the route network actually used by this group of employees, as a subset of the available route network. This may be desirable for analysis purposes, as important route network segments can be distinguished from the unused ones, e.g., when assessing which are acceptable to block during maintenance tasks.

A second quality measure for route network reconstruction is the accuracy of the placement of the reconstructed intersections. Its cumulative error distribution is shown in Figure 8 (left). The figure shows placement deviation from $36 \mathrm{~cm}$ to $30 \mathrm{~m}$, with a median deviation of $3.9 \mathrm{~m}$. This results are comparable with the outdoor results by Fathi [11], who achieve a intersection location accuracy of $4.6 \mathrm{~m}$ when detecting road intersections based on GPS positions. This comparability of results is remarkable in so far as in Fathi's evaluation the positioning accuracy was much higher with around $4 m$, and also the hospitals' indoor route network is denser than outdoor road networks and thus harder to reconstruct without error.

In Figure 8 we illustrate by example the segmentation and mapping of trajectories to network segments, and the resulting benefits not only for reconstruction but also,e.g., for data cleaning and for detection of commonly used routes in the hospital. Despite significant noise, the segmentation algorithm nonetheless produces a proper mapping of sub-trajectories to corridors, here visualized in colors: The green part of the trajectory physically overlaps with the orange part; but this overlap is classified as noise, and not as a real movement between corridors. The pink part shows how the algorithm may performs mapping based on a very small amount of position

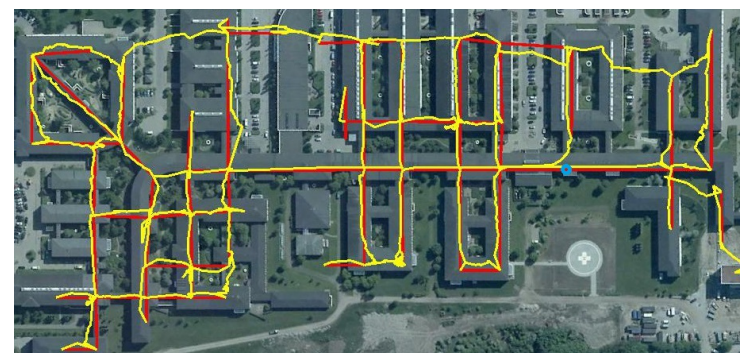

Fig. 9: Median trajectories for indoor data. Map: Google, Aerodata International Surveys

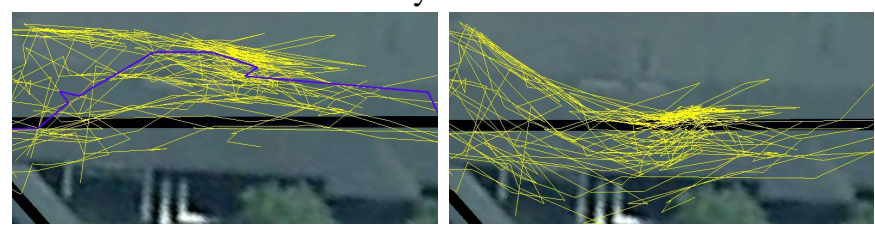

Fig. 10: The deviation map applied to a set of indoor trajectories along a ground-truth corridor (black): Left: trajectories before application (yellow), and median trajectory (blue). Right: trajectories after application.

estimates as obtained at high travel speeds.

\section{B. Representative Trajectories}

In this subsection, we investigate the accuracy of the representative trajectories - on which also both remaining deliverables, the deviation maps and the trajectory cleaning are based on. Figure 9 shows the median trajectories computed for every route network segment. It can be seen how the trajectory segmentation computation supplements the route network reconstruction, and vice versa: a few segments which were yet missing in the reconstructed route network, are now identified, e.g., the connecting corridor between the two parallel north-south-oriented buildings in the north-east corner.

The representative median trajectories for route network segments are much less noisy trajectory representations than the individual trajectories comprising them: Averaged over all route network segments, the sub-trajectories on a given route network segment are on average 1.69 times longer in travel than the median trajectory computed for that segment. We evaluated also the proximity of the median trajectories to their associated segments: Over all route network segments, the associated median trajectories exhibit a median distance of $1.11 \mathrm{~m}$. The is median distance is a less than a mere fourth of the median distance of $4.9 \mathrm{~m}$ that the underlying raw trajectories exhibit. For comparison, when disabling various filters, e.g., clipping, the median distance raises to $1.21 \mathrm{~m}$; for average trajectories, in place of median trajectories, the median distance is $1.22 \mathrm{~m}$.

Some few of the computed median trajectories are significantly noisy or irregular. A comparison with the dataset shown in Figure 6 reveals that these correlate often with the locations where only small amounts of data have been collected. As the median trajectory computations require a representative set of trajectories, this is expected. Of note is also the method's behavior in case an intersection has been missed. Such an example has been marked in the figure by the blue circle. In this case, the resulting median trajectory, oblivious of the intersection, resembles an artificial path curving to the west, but does remain within the physical corridor network. 


\section{Deviation maps and trajectory cleaning}

The local deviation map, given as the distance between the median trajectory and the corridor, can be of use, e.g., for post-hoc analysis of movements, or where to improve the positioning system (here: by placing an access point at the middle of the corridor), or to improve tracking future movement in real-time, e.g., for live navigation purposes. Figure 10 exemplifies the use of deviation maps, focusing on a median shown also in Figure 9: Figure 10 (left) shows the corridor segment (black), the collected trajectories (yellow), and the computed median trajectory (blue). The positioning system's local bias for this corridor (black) is visible in the collected trajectory set (yellow) as well as in the resulting median trajectory (blue). Figure 10 (right) shows the trajectories with their individual positions are corrected according to the deviation map. While many of the original position estimates are biased towards north of the traversed corridor, in Figure 10 (right) the majority of the position estimates have been de-biased and are now much closer to the actual corridor. As a result, the median distance of $4.6 \mathrm{~m}$ between the corridor and the original trajectory shrinks in this example case to a mere $1.8 \mathrm{~m}$. Deviation maps also facilitate the identification of outliers: In presence of a position bias, outliers cannot reliably be identified based solely on the distance from corridors. After applying the deviation maps, however, as the bias has been accounted for, outliers can be identified by their distance to the corridors. Alternatively, the representative trajectories may be used directly, in order to identify outliers relative to them.

\section{CONCLUSions}

We presented novel methods for analyzing and enhancing noisy trajectory sets, specifically from indoor environments, and for reconstructing the route network which underlies the movements represented by a given trajectory set. We evaluated these methods using a large data set of employees' movements within a hospital complex. The evaluation shows that our reconstruction is generally accurate. Secondly, our filter technique for computing median trajectories for movements on individual route parts provides less noisy and more realistic representatives for movements, and it helps revealing local biases in the given positioning system. Finally, we showed how to use the computed information and counter-act said biases by, among others, use of deviation maps.

Future work include the investigation of potential improvements, including a tighter, more iterative interlinking of our methods in order to deal with yet challenging situations such as small and infrequently traveled route network segments. Evaluations in differing environments and settings, e.g., shopping malls, trading fairs, and outdoor spaces, verify the generalisability of the proposed methods.

\section{ACKNOWLEDGMENTS}

The authors acknowledge the support granted by the Danish Advanced Technology Foundation under J.nr. 076-2011-3. Part of this work was done during research visits of the first and third author at University of Münster.

\section{REFERENCES}

[1] M. Alzantot and M. Youssef. Crowdinside: automatic construction of indoor floorplans. In ACM GIS, pages 99-108, 2012.
[2] D. C. Asmar, J. S. Zelek, and S. M. Abdallah. Smartslam: localization and mapping across multi-environments. In Proc. Intl. Conf. Systems, Man and Cybernetics, pages 5240-5245, 2004.

[3] P. Bahl and V. N. Padmanabhan. Radar: An in-building rf-based user location and tracking system. In Proc. IEEE Conf. Computer Communications, pages 775-784, 2000.

[4] J. L. Bentley and T. A. Ottmann. Algorithms for reporting and counting geometric intersections. IEEE Transactions on Computers, C-28(9):643-647, Sept. 1979.

[5] J. Biagioni, T. Gerlich, T. Merrifield, and J. Eriksson. Easytracker: automatic transit tracking, mapping, and arrival time prediction using smartphones. In ACM SenSys, pages 68-81, 2011.

[6] K. Buchin, M. Buchin, M. J. van Kreveld, M. Löffler, R. I. Silveira, C. Wenk, and L. Wiratma. Median trajectories. Algorithmica, 66(3):595-614, 2013.

[7] J.-L. D. Carufel, A. Gheibi, A. Maheshwari, J.-R. Sack, and C. Scheffer. Similarity of polygonal curves in the presence of outliers. Computational Geometry: Theory and Applications, 47(5):625-641, July 2014.

[8] Y.-C. Cheng, Y. Chawathe, A. LaMarca, and J. Krumm. Accuracy characterization for metropolitan-scale wi-fi localization. In MobiSys, pages 233-245. ACM, 2005.

[9] H. Du, P. Henry, X. Ren, M. Cheng, D. B. Goldman, S. M. Seitz, and D. Fox. Interactive $3 \mathrm{~d}$ modeling of indoor environments with a consumer depth camera. In Proc. Intl. Conf. Pervasive and Ubiquitous Computing, pages 75-84, 2011.

[10] H. Durrant-Whyte and T. Bailey. Simultaneous localization and mapping: part i. IEEE Robotics \& Automation Magazine, 13(2):99-110, 2006.

[11] A. Fathi and J. Krumm. Detecting road intersections from GPS traces. In Proc. Intl. Conf. Geographic Information Science, volume 6292 of LNCS, pages 56-69. 2010.

[12] B. Ferris, D. Fox, and N. D. Lawrence. Wifi-slam using gaussian process latent variable models. In IJCAI, volume 7, pages 2480-2485, 2007.

[13] J. Gudmundsson, A. Thom, and J. Vahrenhold. Of motifs and goals: Mining trajectory data. In ACM GIS, pages 129-138, 2012.

[14] J. Huang, D. Millman, M. Quigley, D. Stavens, S. Thrun, and A. Aggarwal. Efficient, generalized indoor wifi graphslam. In Intl Conf. Robotics and Automation (ICRA), pages 1038-1043. IEEE, 2011.

[15] Y. Jiang, Y. Xiang, X. Pan, K. Li, Q. Lv, R. P. Dick, L. Shang, and M. Hannigan. Hallway based automatic indoor floorplan construction using room fingerprints. In Proc. Joint Conf. Pervasive and Ubiquitous Computing, pages 315-324, 2013.

[16] M. B. Kjærgaard, M. V. Krarup, A. Stisen, T. S. Prentow, H. Blunck, K. Grønbæk, and C. S. Jensen. Indoor positioning using Wi-Fi - how well is the problem understood? In Intl. Conf. Indoor Positioning and Indoor Navigation, 2013.

[17] C. N. Klokmose, M. Korn, and H. Blunck. Wifi proximity detection in mobile web applications. In EICS, pages 123-128, 2014.

[18] J.-G. Lee, J. Han, and K.-Y. Whang. Trajectory clustering: A partitionand-group framework. In Proc. ACM SIGMOD Intl. Conf. Management of Data, pages 593-604, 2007.

[19] S. Minamimoto, S. Fujii, H. Yamaguchi, and T. Higashino. Map estimation using gps-equipped mobile wireless nodes. Pervasive and Mobile Computing, 6(6):623-641, 2010.

[20] S. Minamimoto, S. Fujii, H. Yamaguchi, and T. Higashinoz. Local map generation using position and communication history of mobile nodes. In PerCom, pages 2-10, 2010.

[21] D. Philipp, P. Baier, C. Dibak, F. Durr, K. Rothermel, S. Becker, M. Peter, and D. Fritsch. Mapgenie: Grammar-enhanced indoor map construction from crowd-sourced data. In PerCom, pages 139-147. IEEE, 2014.

[22] T. S. Prentow, H. Blunck, K. Grønbæk, and M. B. Kjærgaard. Estimating common pedestrian routes through indoor path networks using position traces. In Proc. Intl. Conf. Mobile Data Management, 2014.

[23] C. E. White, D. Bernstein, and A. L. Kornhauser. Some map matching algorithms for personal navigation assistants. Transportation Research Part C: Emerging Technologies, 8(1):91-108, 2000. 